\title{
Technè
}

La science au service de l'histoire de l'art et de la préservation des biens culturels

$42 \mid 2015$

Science et conservation

\section{Les ambres : approche morphologique des altérations}

Ambers: a morphological approach to alterations

\section{Cécile Giroire, Shéhérazade Bentouati, Dominique Robcis et Juliette Langlois}

\section{OpenEdition}

\section{Journals}

Édition électronique

URL : http://journals.openedition.org/techne/7026

DOI : 10.4000/techne.7026

ISSN : 2534-5168

Éditeur

C2RMF

Édition imprimée

Date de publication : 1 décembre 2015

Pagination : 84-91

ISBN : 978-2-7118-6249-8

ISSN : $1254-7867$

Référence électronique

Cécile Giroire, Shéhérazade Bentouati, Dominique Robcis et Juliette Langlois, « Les ambres : approche morphologique des altérations », Technè [En ligne], 42 | 2015, mis en ligne le 01 décembre 2015 consulté le 11 mars 2021. URL : http://journals.openedition.org/techne/7026 ; DOI : https://doi.org/ 10.4000/techne.7026

\section{(c) (i) (2) $\Theta$}

La revue Technè. La science au service de l'histoire de l'art et de la préservation des biens culturels est mise à disposition selon les termes de la Licence Creative Commons Attribution - Pas d'Utilisation Commerciale - Pas de Modification 4.0 International. 


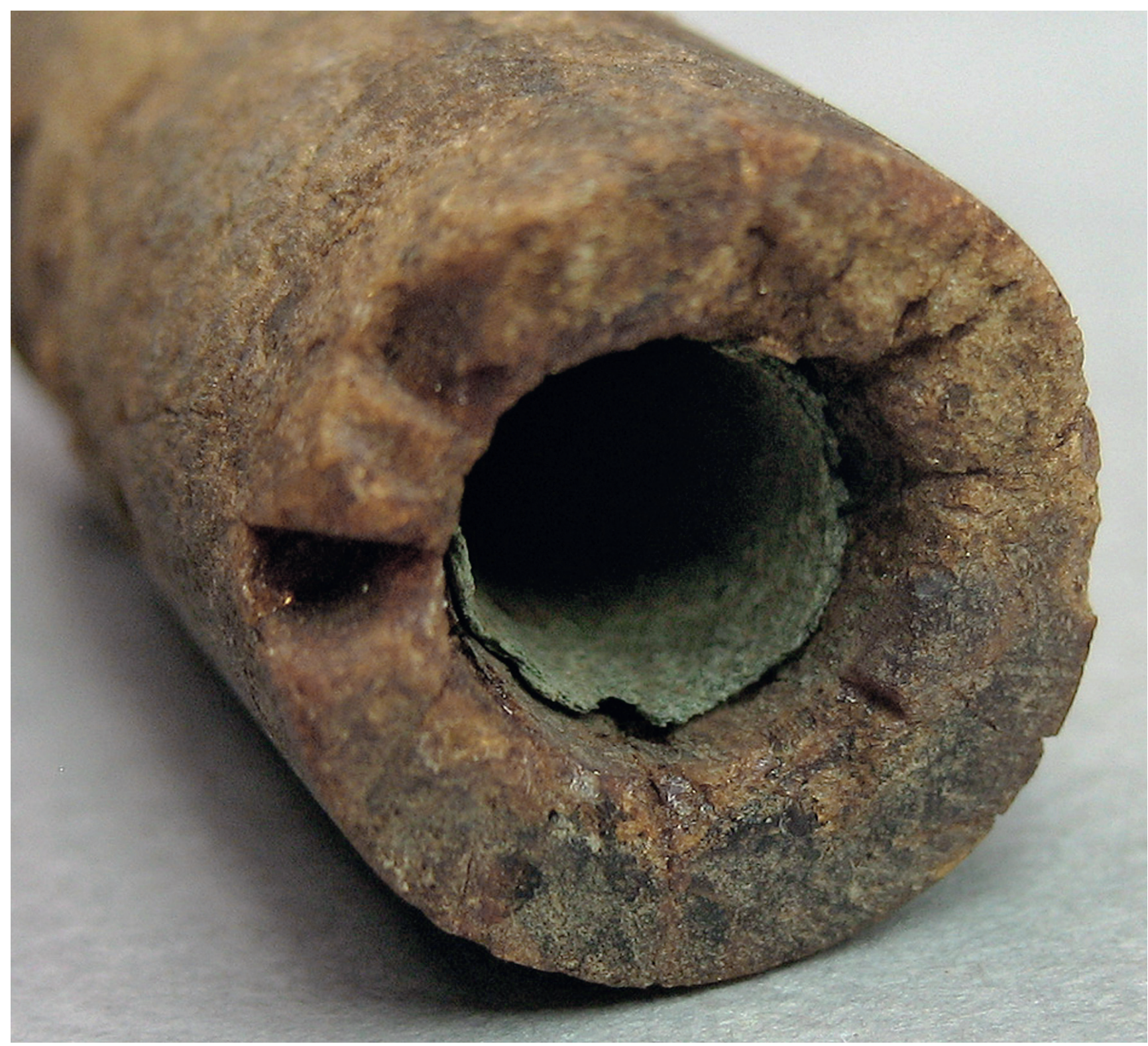

Fig. 1. Feuille métallique, en alliage base cuivre, insérée dans la perforation d'une perle cylindrique, musée du Louvre (Collier Bj 621). @ Shéhérazade Bentouati. 
Cécile Giroire

Shéhérazade Bentouati

Dominique Robcis

Juliette Langlois

\section{Les ambres : approche morphologique des altérations}

Ambers: a morphological approach to alterations

Résumé. Une étude portant sur la collection des ambres conservée au département des Antiquités grecques, étrusques et romaines du musée du Louvre a été réalisée à l'occasion du réaménagement des réserves. De nombreuses informations sur les techniques de fabrication des objets et sur la morphologie de leurs altérations ont été rassemblées et viennent enrichir la connaissance des œuvres. Des examens approfondis ont pu être menés grâce, notamment, à la microscopie numérique.

Mots-clés. Ambre, technique de fabrication, altération, craquelure, fissure, maillage, chromatographie en phase gazeuse, microscopie numérique 3D.
Abstract. A study about the amber collection in the Department of Greek, Etruscan and Roman Antiquities at the Louvre was conducted when the storerooms were refurbished. Information garnered about the manufacturing techniques used on the artefacts and the morphology of their alterations has increased our knowledge of the works. Extensive tests were carried out using digital microscopy.

Keywords. Amber, manufacturing technique, alteration, cracks, fissures, mesh, gas chromatography (GC), 3-D digital microscopy.

\section{Genèse}

Matière emblématique, l'ambre se rencontre dans diverses collections patrimoniales, internationales et européennes, avec notamment celles des musées du Koenigsberg à Berlin, du Nationalmuseet à Copenhague ou encore du British Museum à Londres ${ }^{1}$, ainsi que dans de nombreuses collections italiennes ${ }^{2}$.

À Paris, le département des Antiquités grecques, étrusques et romaines du musée du Louvre conserve également une importante collection d'ambres. Éléments de parures, figurines et blocs gravés constituent le cœur de la collection. De cet ensemble, seules quelques pièces - essentiellement des bijoux - sont exposées au public, la majeure partie étant conservée en réserves.

Pour celles-ci, dans le but d'une conservation optimale, un programme de reconditionnement du corpus a été initié en 2009. C'est lors des différentes manipulations liées à cette opération que le délitement avancé de nombreuses pièces d'ambre a été constaté : effritements et fragmentations sont identifiés comme les altérations récurrentes de l'ambre.

Il a semblé alors nécessaire de revoir la collection dans son intégralité afin de dresser un bilan sanitaire global, de compléter et d'enrichir la connaissance des œuvres, tant sur la typologie et les techniques de fabrication que sur les altérations.

\section{Les origines et les caractéristiques d'une collection}

\section{Provenance des objets et origine géologique de l'ambre}

Issues majoritairement du fonds Campana acquis en 1861, soixante-cinq pièces constituent la collection, dont une vingtaine de natures composites associées à de l'or, de l'argent ou du verre. Leur provenance archéologique et leur datation ne sont pas toujours aisées à établir et le catalogue de De Ridder $^{3}$, ouvrage de référence pour la collection de bijoux du département, ne propose des sites de découverte que pour seulement quelques artéfacts. Une étude récente a permis d'affiner les typologies et de proposer une chronologie. Ainsi, les hypothèses avancées situent la majorité des pièces entre le VIII ${ }^{\mathrm{e}}$ siècle avant J.-C. et le $\mathrm{II}^{\mathrm{e}}$ siècle après J.-C. ${ }^{4}$

Bien souvent, les sites de découverte des ouvres ne correspondent pas à l'origine géologique de l'ambre. En effet, tout comme l'ivoire ou le jais, cette substance végétale a toujours été considérée comme une matière précieuse et souvent utilisée comme monnaie d'échanges. On la rencontre ainsi sous forme de produits finis ou semi-finis, fort loin de son lieu d'extraction.

Cécile Giroire, conservateur du patrimoine, département des AGER, musée du Louvre (cecile.giroire@louvre.fr). Shéhérazade Bentouati, restauratrice du patrimoine métal-ambre (echaraz@yahoo.fr). Dominique Robcis, chef de travaux d'art, département restauration filière archéologique, C2RMF (dominique.robcis@culture.gouv.fr). Juliette Langlois, assistante ingénieur, département Recherche, C2RMF (juliette.langlois@culture.gouv.fr). 
Spontanément, l'ambre est associé aux régions septentrionales de l'Europe et en particulier à la mer Baltique en raison de ses importants gisements ${ }^{5}$. Ce site d'exploitation, le plus connu en Europe, délivre une résine qui est aussi appelée succin en raison de sa forte teneur en acide succinique $^{6}$.

Dans notre contexte d'étude, il n'a pas été envisagé de procéder à une analyse systématique du matériau sachant, en outre, que l'obtention des informations passe par la destruction de l'échantillon ${ }^{7}$. Cependant, lors des premières manipulations, des micro-fragments s'étaient naturellement désolidarisés et ne pouvaient être refixés en raison de l'impossibilité de les localiser. Certains ont de ce fait pu servir à la réalisation d'analyses.

Les biomarqueurs caractéristiques de l'ambre (acide succinique, camphre, (iso)-bornéol notamment), ainsi que des structures abiétanes et pimaranes ont été identifiés grâce à des techniques d'analyses séparatives ${ }^{8}$. Pour les échantillons des cinq pièces étudiées, les profils obtenus sont similaires et correspondent à ceux décrits dans différentes études sur l'ambre de la Baltique (provenant d'une résine issue de la famille des Pinaceae $)^{9}$. L'origine des ambres baltes reste cependant discutée et outre cette variété, il existe plus de soixante autres résines produites dans des conditions analogues en Europe $^{10}$.

\section{L'ambre, naissance d'un polymère}

L'ambre provient d'une oléorésine végétale sécrétée par voies aériennes ou souterraines ${ }^{11}$. La résine est majoritairement composée de terpènes, mais aussi de sève, d'acides et d'huiles ${ }^{12}$. Dès leur formation, les sécrétions s'oxydent et se déshydratent par évaporation des fractions volatiles odorantes. Les fractions non volatiles, qui se manifestent sous forme de substances molles et collantes, durcissent et se stabilisent graduellement sous forme d'ambre par un processus de polymérisation ${ }^{13}$. Lors de la maturation de l'ambre, qui s'étend sur des millions d'années, d'autres processus ont lieu comme l'isomérisation, la réticulation et la cyclisation. Ces modifications s'opèrent sous l'eau en situation anaérobie, et sous certaines conditions élevées de pression et de chaleur ${ }^{14}$. Ce phénomène est d'ailleurs interprété à tort comme une fossilisation. L'ambre ne se fossilise pas, mais subit une modification structurale de ses composés chimiques qui lui confère de nouvelles propriétés ${ }^{15}$.

\section{Le façonnage de l'ambre}

Après son extraction, l'ambre est essentiellement destiné à la fabrication d'éléments de parure. Contrairement aux autres gemmes d'origine minérale, l'ambre est un corps très léger ${ }^{16}$, facile à travailler mais qui reste cependant sensible aux pressions importantes et aux échauffements. Les techniques employées relèvent pour la plupart de celles qui sont mises en œuvre pour le lapidaire ou le travail de l'os. Éléments de fibules ou de colliers, pendentifs et pendeloques sont perforés en vue de leur enfilage. Pour la réalisation des perforations, on suppose l'utilisation d'un outil rotatif de type tour à archet. Ainsi, en observant les parois, on remarque parfois la présence de sillons parallèles. Ce constat n'est pas systématique et d'autres techniques de percement ont été expérimentées telle que l'insertion, directement dans l'ambre, d'une sorte d'alêne métallique chauffée $\mathrm{e}^{17}$.

Sur certaines pièces, on a pu constater la présence de fines feuilles métalliques enroulées et plaquées à l'intérieur de ces perforations (fig. 1) ${ }^{18}$. On suppose une dimension esthétique, l'ambre étant généralement translucide, mais aussi une utilisation en tant que renfort mécanique afin d'éviter l'usure prématurée de l'ambre suite aux frottements répétés. Des examens supplémentaires ont révélé la présence de fibres de bois ou d'empreintes fortement minéralisées sur la face interne des feuilles, ce qui a permis de remettre en cause la typologie de certaines perles de formes cylindriques initialement identifiées comme faisant partie d'un collier ${ }^{19}$. La question du mode de maintien de ces feuilles s'est posée dans un second temps. Il pouvait être uniquement basé sur une flexion mécanique de la feuille ou bien associé à l'utilisation d'un adhésif.

Lors de l'examen de l'une des perles ${ }^{20}$, une fine membrane souple et translucide a été détectée. Cette membrane potentiellement organique a été prélevée, mais sa proximité avec l'ambre n'a pas permis, lors de l'analyse, de caractériser sa nature organique. Cependant, sa localisation coincée entre l'ambre et le métal nous oriente fortement vers l'emploi d'un adhésif préalablement à la mise en place de la feuille (fig. 2a et $2 b)$.

Deux autres pièces de grande qualité ont attiré notre attention. Il s'agit d'un masque théâtral figurant Héraclès et d'une petite lampe, respectivement fig. 3 (H. 5,8 x 1. 6,3 cm) et fig. $4(1.8,2 \times \text { D. } 5,2 \mathrm{~cm})^{21}$. Pour ces deux objets, des blocs d'ambre suffisamment importants et homogènes ont été utilisés. Les volumes ont été " taillés » dans la masse et les deux pièces présentent des parties évidées. Pour le masque d'Héraclès, c'est à nouveau un outil rotatif qui a été employé. Dès l'âge du fer, le tour horizontal est utilisé pour la fabrication de petits ornements ${ }^{22}$. Cependant, les parois ne sont marquées de stries que jusqu'à mi-hauteur et le profil exact de l'outil reste encore à déterminer. Sur la lampe, l'absence totale de traces d'outils laisse en suspens nos multiples interrogations.

Ces deux objets monobloc confirment une véritable mâ̂trise par les artisans des techniques de fabrication et témoignent d'une incontestable connaissance de la matière. 


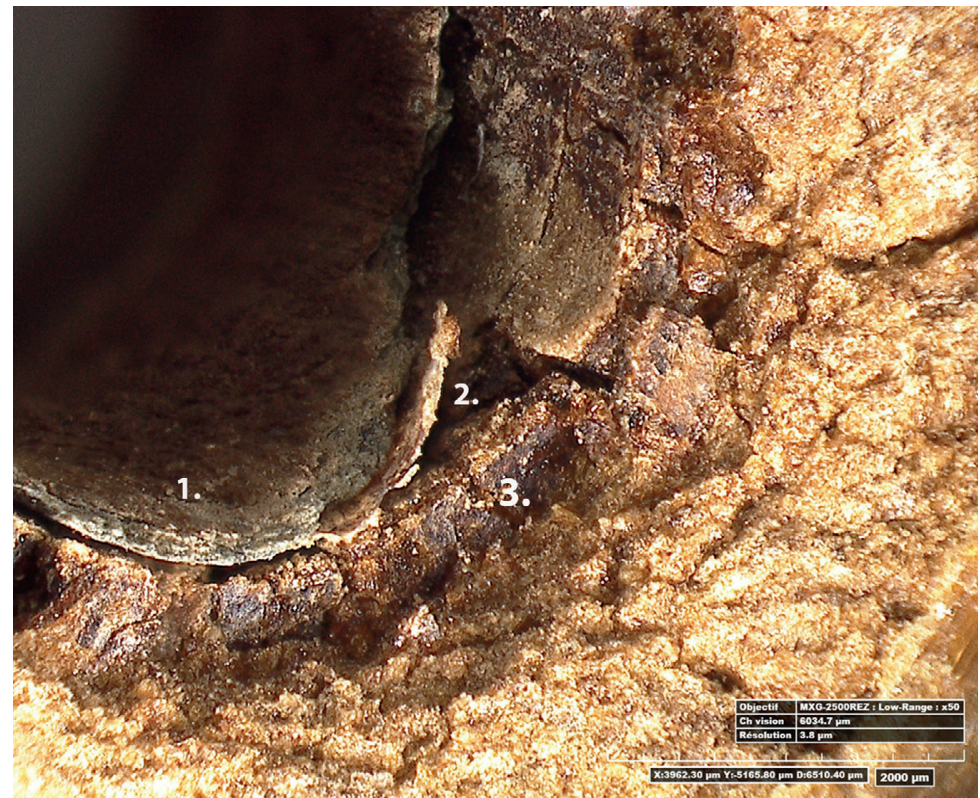

Fig. 2a. Membrane souple et translucide plaquée contre la feuille de métal et l'ambre (microscope numérique 3D, x 50). 1 : feuille métallique. 2 : membrane adhésive ? 3 : ambre. (C) C2RMF/Dominique Robcis.

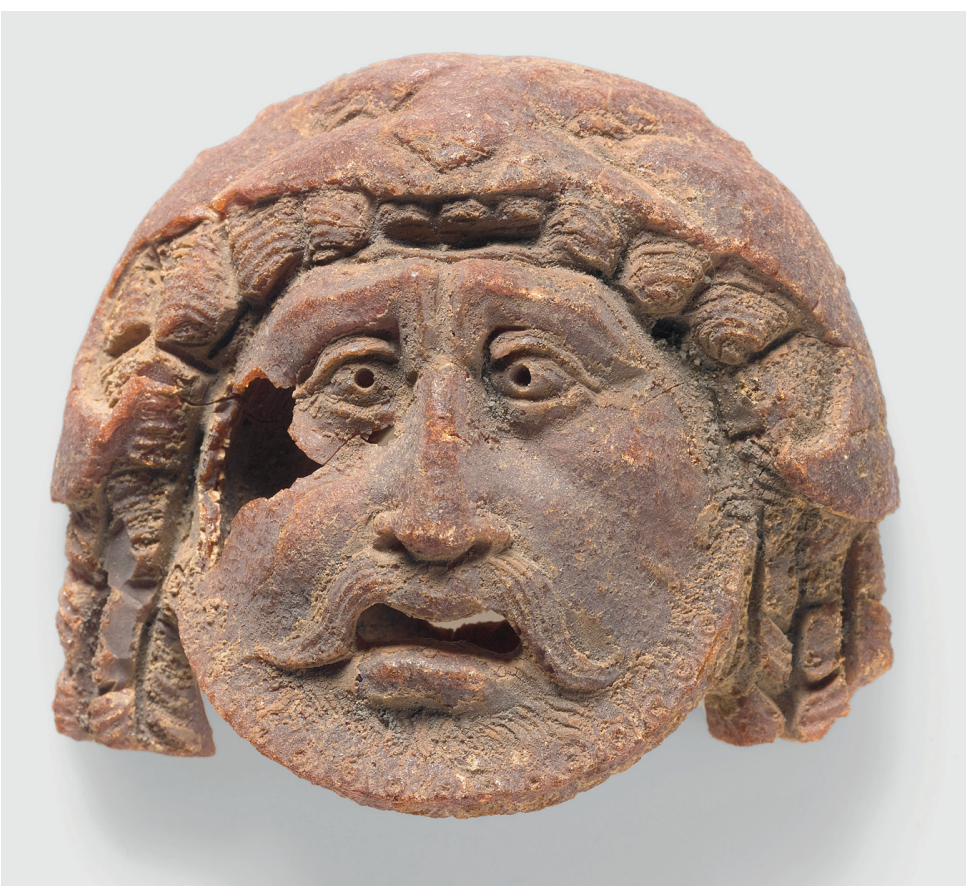

Fig. 3. Masque théâtral : Héraclès (Bj 2121). I ${ }^{\mathrm{er}}$ siècle apr. J.-C.,

provenance inconnue (H. $5,8 \mathrm{~cm} \times 1.6,3 \mathrm{~cm})$. ๑ RMN-Grand Palais (musée du Louvre)/Hervé Lewandowski.

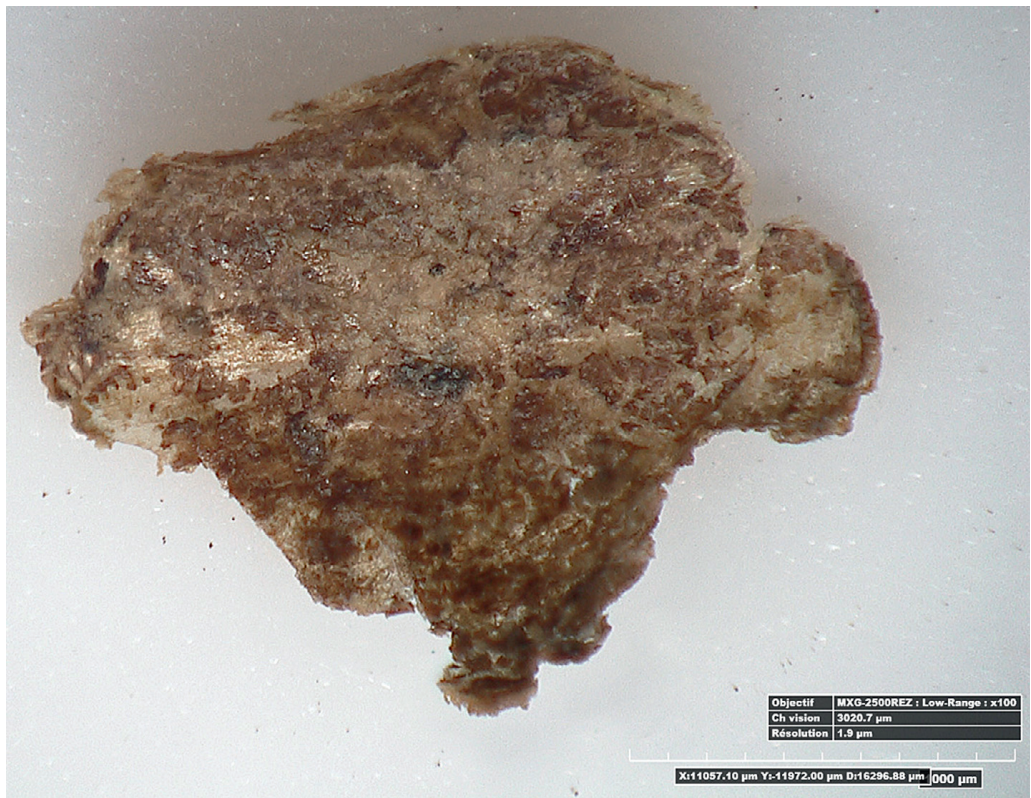

Fig. 2b. Visualisation de la membrane après prélèvement

(microscope numérique 3D, x 50). ( $) \mathrm{C} 2 \mathrm{RMF} /$ Dominique Robcis.

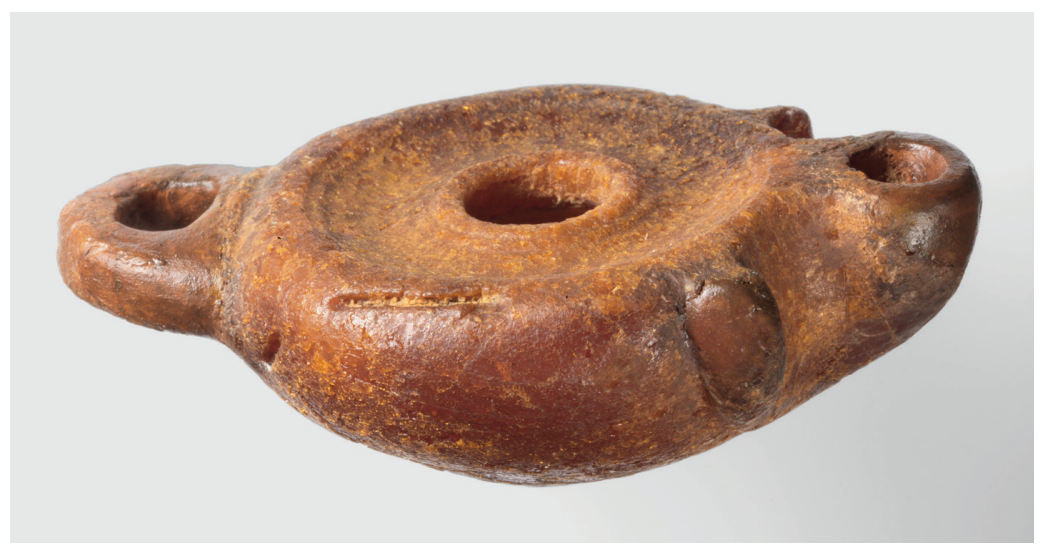

Fig. 4. Lampe (inv. Bj 2149). $\mathrm{I}^{\mathrm{er}}$ siècle apr. J.-C., provenance inconnue (H. 2,6 cm x l. 8,2 cm). @ Musée du Louvre, Dist. RMN-Grand Palais/ Philippe Fuzeau. 


\section{Morphologie des altérations}

\section{Les facteurs d'altérations}

Dès son extraction, l'ambre se dégrade sous l'action de quatre principaux facteurs environnementaux : l'humidité relative, l'oxygène, la température et la lumière ${ }^{23}$. Des études ont permis de mettre en évidence des processus de dépolymérisation, comme l'hydrolyse et l'oxydation, entraînant la dégradation de la structure chimique ${ }^{24}$. L'hydrolyse en milieux acide et basique conduit à la formation d'acide succinique et de communol. Les milieux alcalins provoquent l'apparition de phénomènes de saponification directement liés au délitement et à la fissuration. En tout état de cause, l'oxydation initiée en surface des composés terpéniques est le processus le plus dommageable. La dégradation des monomères, avant polymérisation et après dépolymérisation, produit également des composés organiques volatiles dont l'émanation augmente sous atmosphère sèche. L'ambre est donc continuellement modifié par son environnement avec, à chaque stade, une évolution de sa structure moléculaire.

\section{Altérations structurelles}

Les altérations structurelles de l'ambre se traduisent principalement par l'apparition de multiples fentes de retrait dues à la dessiccation. Leur concentration en surface provoque une perte du poli d'origine et, en modifiant la réflexion de la lumière, un assombrissement général qui affecte grandement les colorations. Dans la majorité des cas, les objets présentent une couche totalement opacifiée et mate en surface. Les éclats et les tranches cassées rendent parfois possible l'observation de couches internes superposées qui présentent des faciès différents avec, pour la plupart, un noyau brillant et translucide à cour (couche moins altérée) .

Dans le cadre de ce travail, des examens approfondis ont été réalisés sous loupe binoculaire et en microscopie numérique $^{25}$. À l'aide de ces équipements, les caractéristiques et la répartition des altérations ont été explorées. Ainsi, les morphologies observées sont très variées avec la formation de craquelures et de fissures multidirectionnelles, de dimensions macroscopiques et microscopiques. Leur mode de propagation est entièrement soumis à la microstructure du matériau. Ces craquelures et ces fissures s'organisent en réseaux qui se développent parallèlement ou perpendiculairement à la surface et se répartissent au sein de strates nettement délimitées.

L'examen d'une coupe d'un ambre moderne a permis de comprendre la genèse de ces strates qui résultent de l'écoulement initial de la résine avec la formation successive d'ondes de mouvement.

Lors de son exsudation, et avant de se détacher de l'arbre, une coulure de résine se superpose à une autre ${ }^{26}$. Le faible degré d'altération de l'ambre moderne a permis de cerner

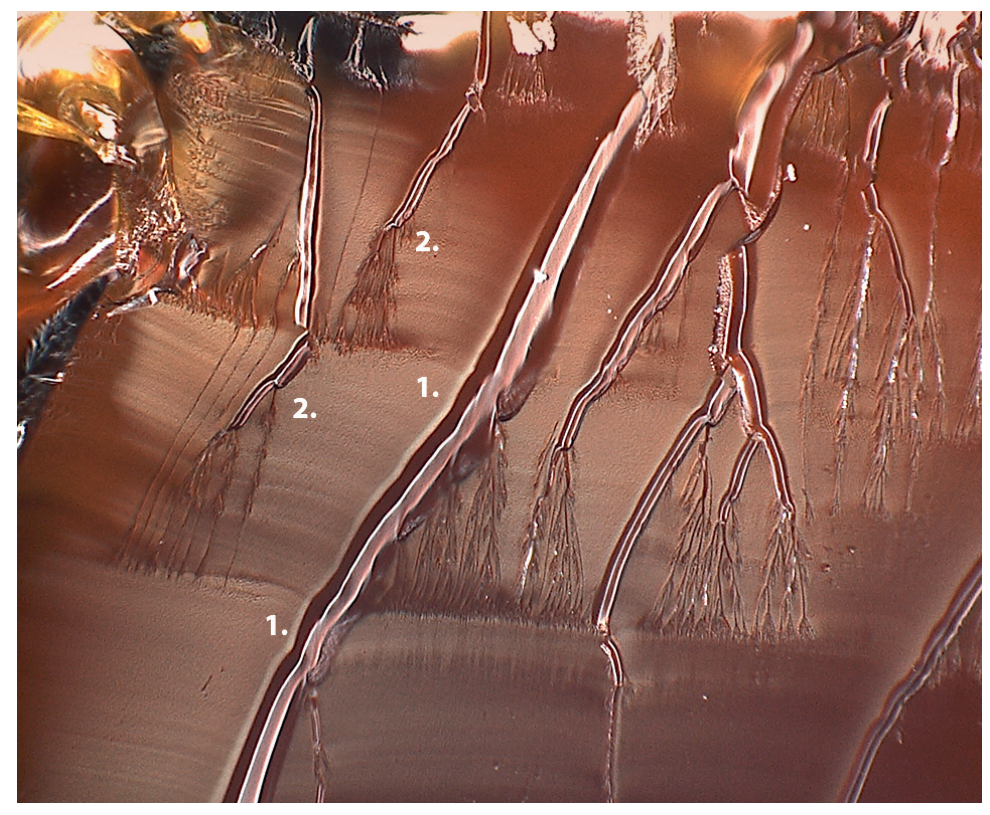

Fig. 5. Vue en coupe d'un ambre moderne (microscope numérique 3D, x 200). 1 : plis formés par les coulées successives de résine. 2 : interruptions ou éclatements des fissures. (C) C2RMF/Dominique Robcis.

avec netteté cette conformation, qui a une véritable incidence sur le développement des fissures. En effet, entre chaque coulée, des paliers se créent et provoquent des ruptures de trajectoire lorsque la fente rencontre ce seuil. Elle peut s'arrêter ou se subdiviser en un ou plusieurs faisceaux. Cet éclatement permet la répartition des contraintes liées au changement de direction. Il soulage notablement la fissure principale mais augmente d'autant le nombre de microfissures et de vide dans la matière (fig. 5). Outre les changements de strates, la trajectoire et les caractéristiques d'une fissure peuvent aussi être modifiées par la présence de bulles d'air (fig. 6b).

Sur les ambres archéologiques, les surfaces présentent de nombreuses microrugosités et, à l'examen microscopique, apparaît un maillage serré d'enchevêtrements de fissures qui finissent par transformer la matière en un conglomérat granuleux qui augmente les porosités et multiplie les réactions de surface (fig. 6a). Dans les sous-couches visiblement plus lisses et brillantes, les fissurations sont davantage espacées (fig. 7).

D’autres pièces révèlent de nombreuses microfissures qui se sont développées en réseaux craquelés sur une épaisseur infime de l'épiderme lequel, suivant son degré de saturation, finit par se détacher en feuillets et rend les couches superficielles friables.

À l'intérieur de ces réseaux craquelés, deux types de maillage se côtoient : un primaire et un secondaire qui se déploie à l'intérieur du premier (fig. 8a et 8b).

La mesure des mailles révèle une certaine régularité. Ainsi, les largeurs des « grains » qui constituent le maillage primaire oscillent entre 1000 et $2000 \mu \mathrm{m}$ (fig. 8a). Ces 


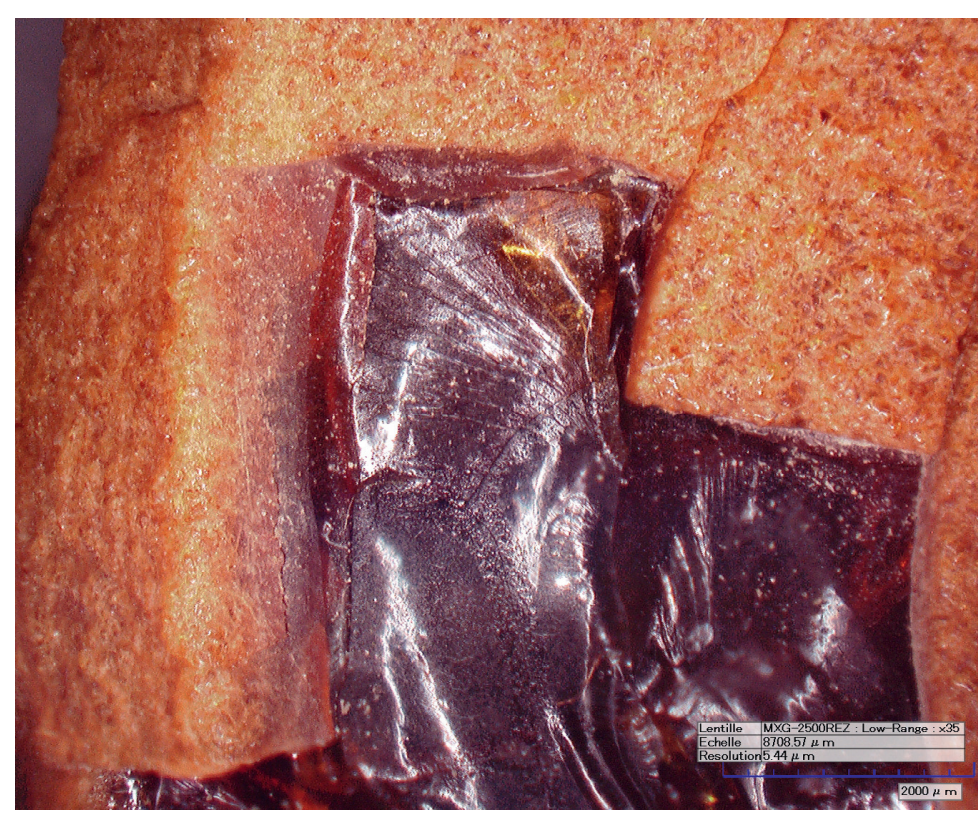

Fig. 6a. Répartition des strates. Couche externe opaque et mate devenant translucide et brillante vers l'intérieur (microscope numérique 3D, x 35). (C C2RMF/Dominique Robcis.

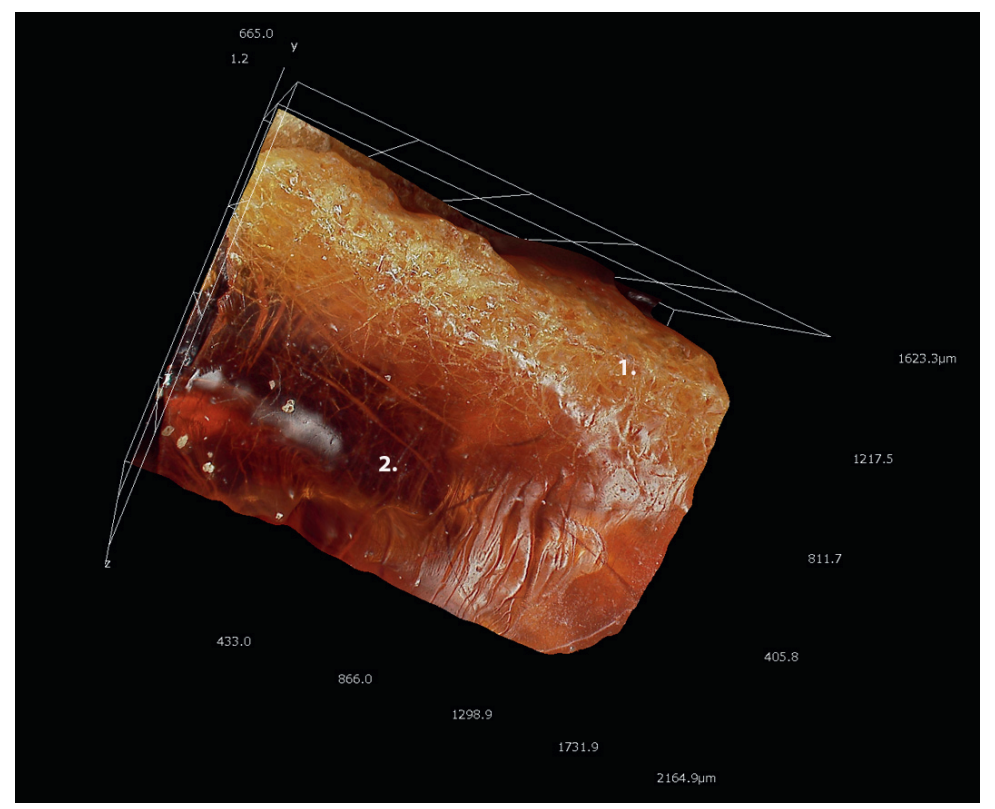

Fig. 7. Répartition et densité du réseau fissuré (microscope numérique 3D). 1 : couche externe. 2 : couche interne. (c) C2RMF/Dominique Robcis.

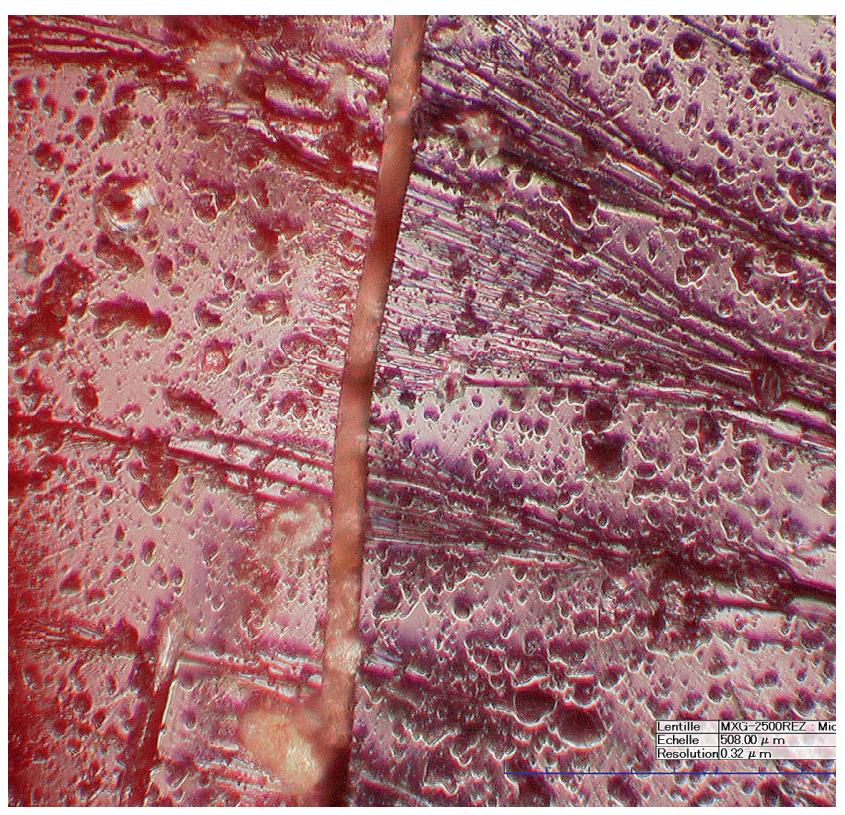

Fig. 6b. Fissurations et cavités microscopiques (microscope numérique 3D, éclairage coaxial, x 600). () C2RMF/Dominique Robcis.

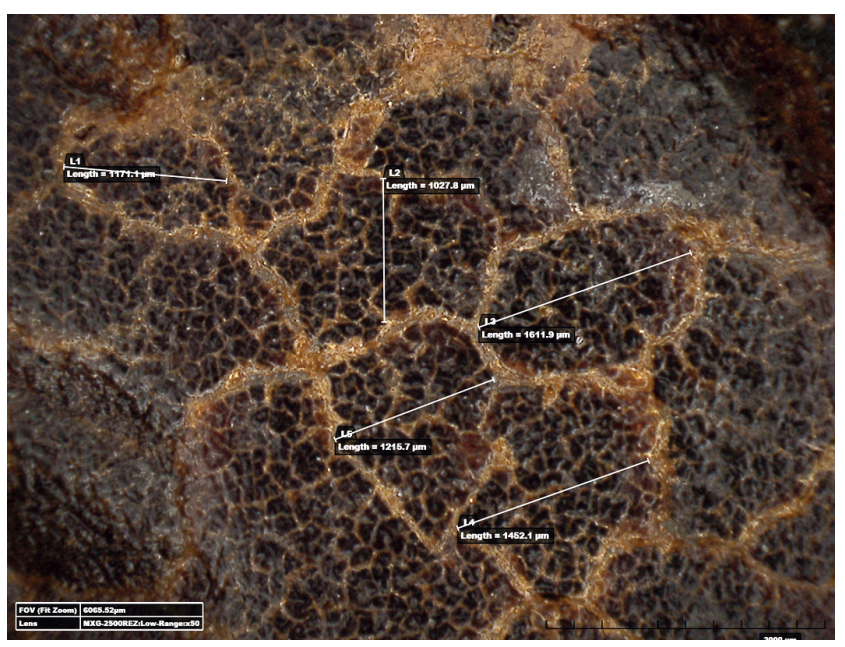

Fig. 8a. Réseau dense de craquelures sinueuses formant des polygones plus ou moins réguliers et mesure du réseau primaire (Tête de bélier, musée du Louvre, Bj 2130) (microscope numérique 3D x 50). (C) C2RMF/Dominique Robcis.

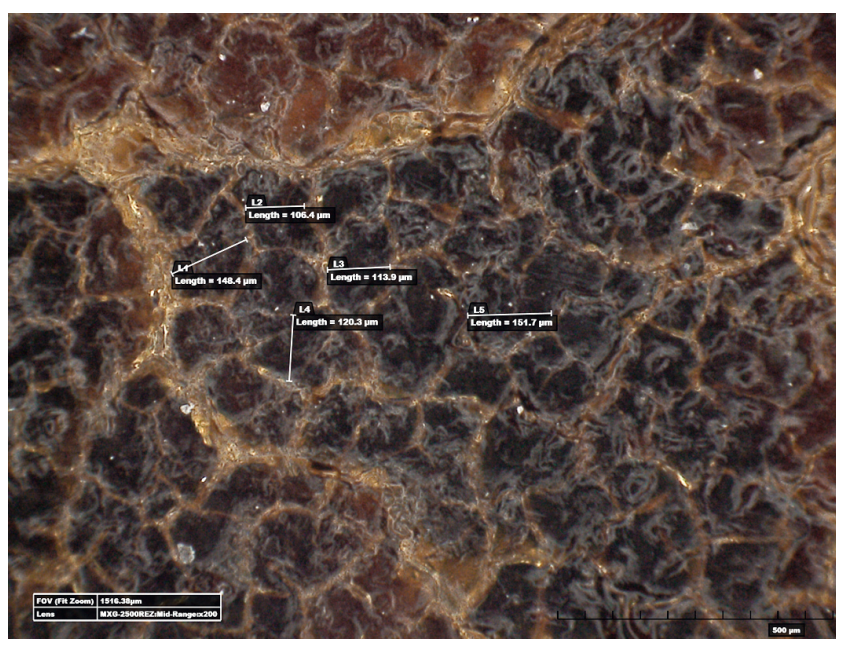

Fig. 8 b. Mesure du réseau secondaire inscrit à l'intérieur du réseau primaire aperçu en fig. $8 \mathrm{a}$ (Tête de bélier, musée du Louvre, Bj 2130) (microscope numérique $3 \mathrm{D} \times 200)$

(c) C2RMF/Dominique Robcis. 


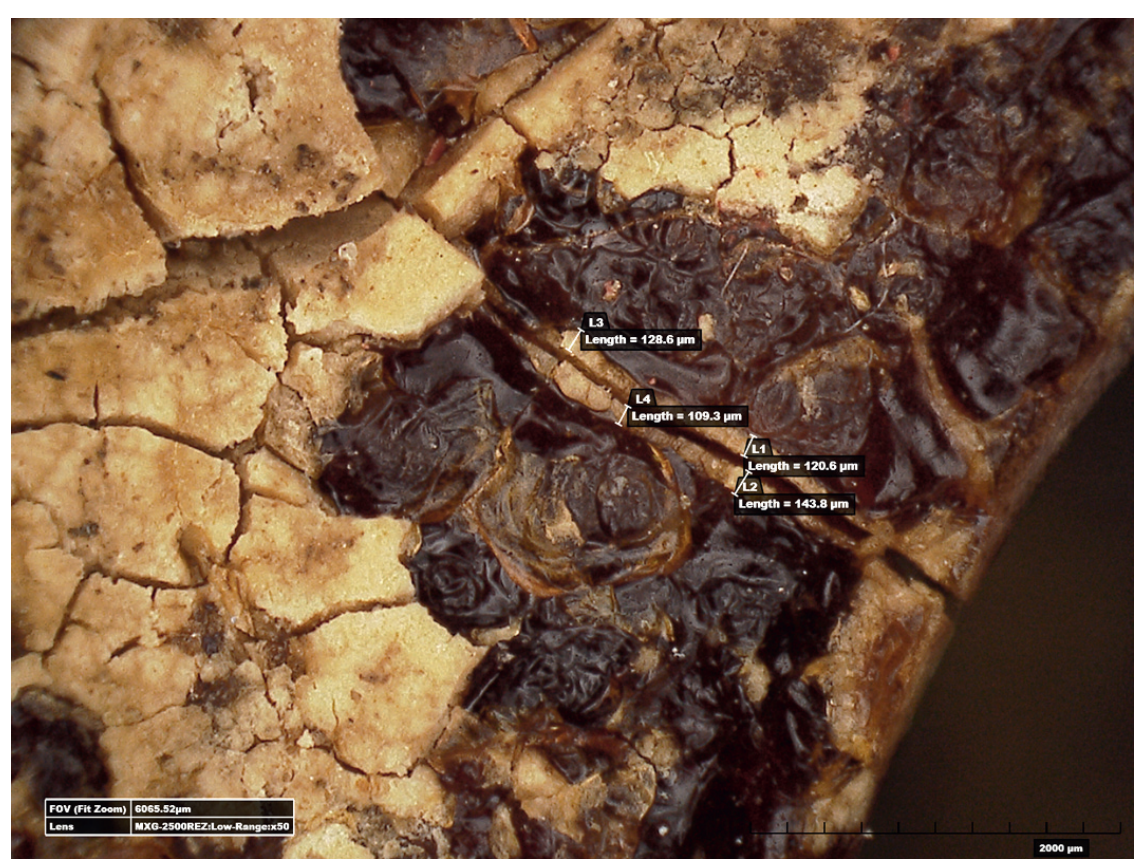

Fig. 9. Par recoupement des réseaux, formation de grains situés entre 100 et $200 \mu^{2}$ fragmentant les surfaces (Masque d'Héraclès, Bj 2121) (microscope numérique 3D x 50). (C) $\mathrm{C} 2 \mathrm{RMF} /$ Dominique Robcis

dimensions ont également été observées sur l'ambre moderne. Sur le maillage secondaire, les mesures s'étirent entre 100 et $200 \mu \mathrm{m}$ (fig. 8b). Cette répartition se répercute sur toutes les surfaces libres en contact avec l'environnement. En conséquence, lorsque les mailles se recoupent, des grains de 100 à $200 \mu^{2}$ peuvent se former, augmentant la porosité des couches et conduisant à terme à son effritement (fig. 9). Un fait est à noter cependant : malgré l'observation en microscopie de nombreuses discontinuités sous forme de cavités ou de fracturations, les pièces d'ambre conservent une certaine cohésion. Ce phénomène doit être relié en partie aux différentes interactions électrostatiques attractives intrinsèques au matériau.

En dernier lieu, on remarque que le tracé des fissures est marqué par une coloration blanchâtre. Cet état spécifique est en partie lié à la diffusion de la lumière, mais également à l'accumulation aux abords des fentes de composés non volatiles véhiculés lors de l'évaporation des fluides. Sur le masque d'Héraclès, objet fortement altéré, on relève la présence de ce type d'amoncellements en surface.

\section{Conclusion}

Cette approche globale de la collection des ambres du département des Antiquités grecques, étrusques et romaines a permis de remettre en question la typologie de certaines pièces, d'enrichir les connaissances sur les techniques de mise en ouvre et sur les altérations spécifiques de ce matériau.

L'utilisation de tours pour évider les pièces et pour les perforer semble être récurrent et l'emploi fréquent de feuilles métalliques à l'intérieur des perforations est attesté sur de multiples objets. Néanmoins, l'étendue des techniques utilisées pour leur fabrication demande à être affinée.

D'un point de vue physico-chimique, nous avons pu aborder les mécanismes successifs de polymérisation et de dépolymérisation de l'ambre qui engendrent l'essentiel des altérations structurelles. Sur ce point, la microscopie numérique $3 \mathrm{D}$ a joué un rôle essentiel. Nous avons ainsi pu visualiser les multiples hétérogénéités structurelles qui fragilisent le matériau et les examens ont permis de distinguer deux principaux modes de formation des fissures, en réseaux parallèles et en réseaux perpendiculaires à la surface. Le réseau parallèle correspond aux coulées apparues lors de l'exsudation de la résine et le réseau perpendiculaire doit être rapproché des chemins pris lors de l'évaporation de certains composés. Au sein de ces réseaux, on a pu constater que les craquelures s'organisent sous forme de maillages, primaires et secondaires, hiérarchisés selon leurs dimensions.

Le recoupement de ces réseaux et de ces mailles conduit à la fragmentation de la matière à l'échelle millimétrique. Ce schéma semble se reproduire également à l'échelle micrométrique. La similitude des mesures de mailles de craquelures, effectuées tant sur des ambres altérés que modernes, a mis en évidence des faciès identiques d'altérations. Même si les mécanismes chimiques ne sont pas encore pleinement explicités, cette caractérisation morphologique permet de mieux comprendre les différents degrés d'altération de la matière. Elle est la première étape avant la mise en place d'un protocole de restauration où les traitements choisis doivent correspondre à la nature du matériau, à son état de surface et à son état structurel. Pour certaines pièces d'ambre, il semble maintenant 
évident qu'une simple consolidation de surface ne suffit pas à garantir leur intégrité et qu'il faut plutôt envisager des traitements d'imprégnation «à cœur » et s'orienter vers des consolidants de très basse viscosité.

En parallèle, des mesures de conservation préventive sont nécessaires afin d'assurer la pérennité de ces œuvres. Les facteurs d'altération de l'ambre regroupant la quasi-totalité des facteurs environnementaux, les mesures de conservation s'avèrent drastiques et il s'agira de trouver des compromis entre la préservation des œuvres et leur exposition au public. Ces questions essentielles sont pour le moment à l'étude concernant la collection des ambres du département des Antiquités grecques, étrusques et romaines.

Notes

1. Strong, 1966.

2. Nava \& Salerno, 2007.

3. De Ridder, 1924.

4. Cette collection a été récemment étudiée et publiée : D'Ercole, 2013.

5. Pline l'Ancien précise déjà dans ses écrits que la principale source de l'ambre utilisée à l'époque romaine en Italie est l'ambre de la Baltique. Voir Pline l'Ancien, Histoire naturelle, XXXVII, 11, 11-13.

6 . Il représente alors 3 à $8 \%$ de la masse globale.

7. Les micro-prélèvements destinés à la GCMS et la Py-GCMS sont de l'ordre de $100 \mu \mathrm{m}$.

8. Chromatographie en phase gazeuse couplée à la spectrométrie de masse (GCMS) et pyrolyse, puis chromatographie en phase gazeuse (py-GCMS. C2RMF, rapport d'analyses 25258 par Juliette Langlois.

9. Czechowski et al., 1996, Yamamoto et al., 2006.

10. Krzeminska, 1992

11. Krzeminska, 1992.

12. Geirnaert, 2002.

13. L'ambre est composé d'un mélange de molécules. Ceci est le cas de toutes les substances naturelles d'origine végétale ou animale.

14. Santiago-Blay et Lambert, 2007.

15. La fossilisation est un processus de substitution d'une matière par une autre avec une modification de la constitution chimique. Geirnaert, 2002.

16. Densité : 1,05 à 1,1 .

17. Du Gardin, 1986.
18. Sur les perles cylindriques du collier Bj 621, les disques de fibules Bj 2245 et 2246. L'alliage employé est une base cuivre aujourd'hui fortement minéralisé.

19. D'Ercole, 2013, p. 88.

20. Collier Bj 621.

21. Respectivement Bj 2121 et Bj 2149.

22. Minni, 2003.

23. Mills, 2011.

24. La variété étudiée est l'ambre

de la Baltique. Pastorelli, 2009

25. Les observations ont été effectuées

au C2RMF avec un appareil Hirox KH-8700.

26. Geirnaert, 2002.

\section{Bibliographie sélective}

Baudrimont M., 1864, «Du soufre considéré comme l'un des éléments constitutifs du succin ", Bulletin de la société chimique, janvier-juin 1864.

D'Ercole M. C., 2013, Ambres gravés. La collection du département des Antiquités grecques, étrusques et romaines, Paris.

De Ridder A., 1924, Catalogue sommaire des bijoux antiques. Musée national du Louvre. Département des Antiquités grecques et romaines, Paris.

Du Gardin C., 1986, « La parure d'ambre à l'âge du Bronze en France ", Bulletin de la Société préhistorique française, tome 83, N. 11-12, p. 546-588.

Geirnaert E., 2002, L'ambre, miel de Fortune et Mémoire de vie, éd. du Piat, Monistrolsur-Loire.

Grimaldi D. A., 1996, Amber: Windows to the Past, The American Museum of Natural History \& Abrams, New York.
Krzeminska E., 1992, Les fantômes de l'ambre : insectes fossiles dans l'ambre de la Baltique, Musée d'histoire naturelle, Neuchâtel.

Mills J. et White R., 2011, The organic chemistry of museum objects, $2^{\mathrm{e}}$ édition, Routledge.

Minni D., 2003, « Le tournage du bois, des lignites, de l'ambre et des matières dures d'origine animale à l'Age du fer ", Le tournage des origines à l'an Mil. Actes du colloque de Niederbronn, éd. Monique Mergoil, p.114.

Nava M. L. et Salerno A., 2007, Ambre. Transparenze dall'Antico. Cat. exp. Naples, Museo Archeologico Nazionale.

Pline l'Ancien, Histoire naturelle, Livre IV, traduit et commenté par Ernout A., éd. Les Belles Lettres, 1952, Paris.

Santiago-Blay J. et Lambert J., 2007, «Aux sources de l'ambre », Pour la science, $\mathrm{n}^{\circ} 356$, juin 200, p. 70-75.

Strong D., 1966, Catalogue of the Carved Amber in the Department of Greek and Roman Antiquities, Londres.

Yamamoto S., Otto A., Krumbiegel G. et Simoneit B.R.T., 2006, "The natural product biomarkers in succinite, glessite and stantienite ambers from Bitterfeld, Germany", Review of Palaeobotany and Palynology, 140 , p. 27-49.

Documents inédits

Pastorelli G., 2009, Archaeological Balticamber: Degradation mechanisms and conservation measures, Dottorato di Ricerca, Università di Bologna. 